\title{
Skeletal dysplasia-epilepsy-short stature syndrome
}

INSERM

\section{Source}

INSERM. (1999). Orphanet: an online rare disease and orphan drug data base. Skeletal dysplasia-epilepsy-short stature syndrome. ORPHA:1858

Skeletal dysplasia-epilepsy-short stature syndrome is characterized by moderate to severe intellectual deficit, seizures, short stature, and skeletal dysplasia. It has been described in seven patients. Other manifestations can be associated (retinal abnormalities, brachydactyly, prognathism, dental malocclusion). It is transmitted as an autosomal recessive trait. 